\title{
Neostigmine Treatment of Paralytic Ileus in Critically Ill Patients
}

\author{
Nak-Jun Choi, M.D., Tae-Hyun Kim, M.D., Suk-Kyung Hong, M.D., Ph.D. \\ Division of Trauma and Surgical Critical Care, Department of Surgery, \\ Asan Medical Center, University of Ulsan College of Medicine, Seoul, Korea
}

\section{Correspondence to:}

Suk-Kyung Hong, M.D., Ph.D.

Division of Trauma and Surgical

Critical Care, Department of

Surgery, Asan Medical Center,

88 Olympic-ro 43-gil,

Songpa-gu, Seoul 05505, Korea

Tel: +82-2-3010-6515

Fax: +82-2-3010-6516

E-mail: skhong94@amc.seoul.kr
Paralytic ileus is a common complication in critically ill patients, and can affect all parts of the gastrointestinal tract. We report a case of paralytic ileus that improved after neostigmine administration. An 80-year-old man was transferred to the intensive care unit after a diagnosis of severe colitis due to Clostridium difficile infection while under conservative treatment for paralytic ileus. The patient's colitis resolved but the ileus did not. This prompted neostigmine administration, resulting in remarkable improvements in the abdominal physical examination and radiographic images. Bowel movements recovered, oral feeding was restarted, and the patient was transferred back to the general ward. (J Acute Care Surg 2017;7:78-82)

Key Words: Paralytic ileus, Critical illness, Neostigmine

Received October 13, 2016, Revised July 7, 2017, Accepted July 24, 2017

Copyright $\odot 2017$ by Korean Society of Acute Care Surgery

(c) This is an Open Access article distributed under the terms of the Creative Commons Attribution Non-Commercial License (http://creativecommons.org/licenses/by-nc/4.0) which permits unrestricted non-commercial use, distribution, and reproduction in any medium, provided the original work is properly cited.

\section{Introduction}

Paralytic ileus may affect all parts of the gastrointestinal tract and is a common serious complication in critically ill patients. It is characterized by abdominal distension, lack of bowel sounds, accumulation of gas and fluids in the bowel, and decreased gastrointestinal passage with delayed or absent defecation. Ileus in critically ill patients prohibits enteral feeding and may lead to increased intra-abdominal pressure (IAP). It also promotes bacterial overgrowth and translocation, as well as absorption of endotoxins [1].

Neostigmine is an acetylcholinesterase inhibitor that increases cholinergic (parasympathetic) activity in the intestinal wall, which in turn stimulates colonic motility. Nevertheless, the clinical utility of neostigmine may be limited by its adverse effects, which include abdominal cramps, excessive salivation, vomiting, and bradycardia [2]. Neostigmine is clinically indicated for myasthenia gravis, reversal of action of muscle relaxants, arousal from anesthesia in operating rooms, or postoperative urinary retention [3]. Having encountered a patient with persistent paralytic ileus unresponsive to conservative treatment, we report this case of a patient with paralytic ileus for whom neostigmine administration was effective.

\section{Case Report}

An 80-year-old man was admitted to this medical center through the Urology Outpatient Department with the chief complaint of voiding difficulty. A Foley catheter had been inserted at another hospital, before he was transferred to this medical center. Two months before admission, he was treated for sepsis 
secondary to repeated urinary tract infection at a local hospital. The patient also had a medical history of hypertension, diabetes mellitus, arrhythmia, and prostatic hypertrophy, as well as subtotal gastrectomy due to gastric ulcer 20 years previously. He had no limitations in performing activities of daily living.

On the fourth day of admission, the patient experienced abdominal pain and bilious vomit of about $500 \mathrm{ml}$. Abdominal plain radiography at that time revealed small bowel ileus. Abdominal computed tomography (CT) carried out the next day confirmed a mechanical obstruction caused by a postoperative adhesive band. The patient was treated conservatively, including nil per oral (NPO), decompression by nasogastric tube (L-tube), and fluid infusion. He had a fever of $38.2^{\circ} \mathrm{C}$ and continued diarrhea without symptomatic improvement. In addition, his ileus did not resolve (Fig. 1). On the 13th day of admission, his blood pressure was $80 / 50 \mathrm{mmHg}$ and heart beat was 140 beats/min, with an altered mental state. After intubation, he was transferred to the surgical intensive care unit (SICU) and placed on mechanical ventilation. Clostridium difficile was identified in his stool culture and polymerase chain reaction. Severe colitis of the sigmoid colon was confirmed on the abdominal CT performed after transfer to SICU (Fig. 2). A diagnosis of pseudomembranous colitis was made. Intravenous (IV) metronidazole was initiated and used for 2 weeks. The previously seen postoperative adhesive band was no longer observed, but paralytic ileus with overall expansion

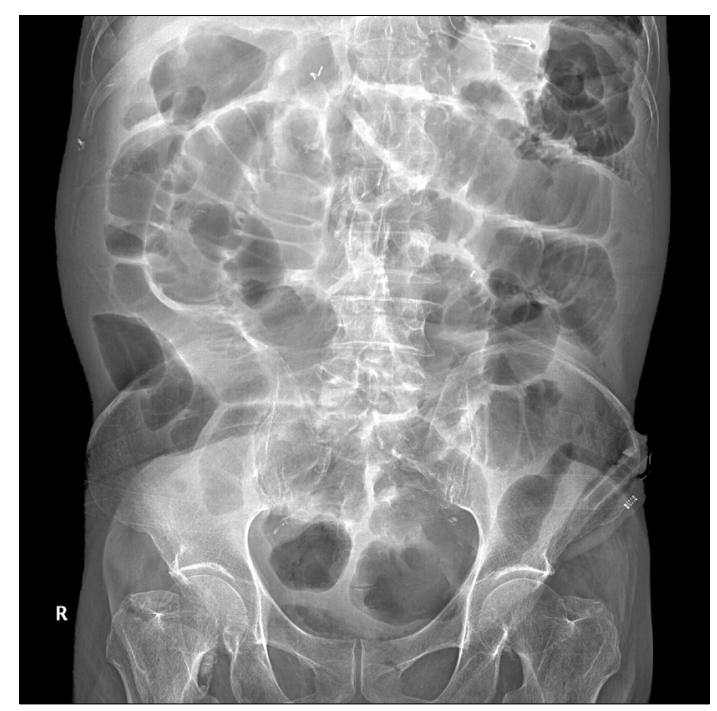

Fig. 1. Abdominal plain radiogram of paralytic ileus. of the small bowel was verified.

A laboratory study carried out eight days after IV metronidazole treatment confirmed negative C. difficile, and follow-up CT images showed that the colitis had largely resolved. He recovered, without the use of vasopressors, from the shock at the time of transfer to SICU. The patient's blood pressure and pulse were maintained at 110/50 $\mathrm{mmHg}$ and 80 beats/min, respectively. The C-reactive protein level, which is indicative of an inflammatory process, reduced from $17.04 \mathrm{mg} / \mathrm{dl}$ to $2.41 \mathrm{mg} / \mathrm{dl}$. This shows that the patient's pseudomembranous colitis was clinically resolved. Nevertheless, he continued to have ileus that did not respond to conservative treatment. Therefore, $2 \mathrm{mg}$ neostigmine was administered in $50 \mathrm{ml}$ normal saline infused over 30 minutes. The patient's condition was observed through monitoring during and after neostigmine administration. A physical examination performed immediately after the drug infusion showed a slightly softer abdomen on palpation and increased bowel sounds on auscultation in comparison with before neostigmine administration. No particular adverse effects were observed. The next day, a further dose of neostigmine was infused with a similar pharmacologic response. Excessive salivation lasting about four hours was observed; besides this, no other adverse effects were observed. Abdominal plain radiography performed before (Fig. 3) and after neostigmine administration (Fig. 4) revealed remarkable amelioration of the ileus as intestinal gases were discharged.

Abdominal palpation performed thereafter revealed a much

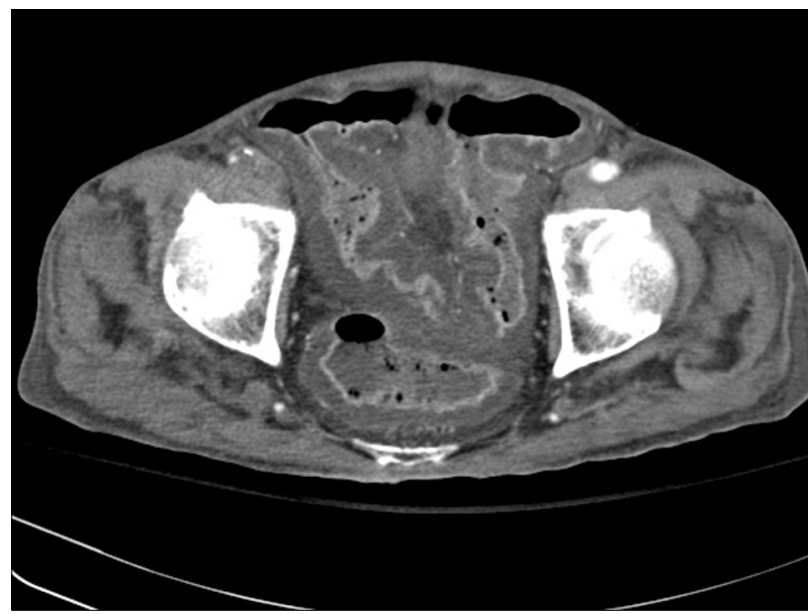

Fig. 2. An abdominopelvic computed tomography with enhancement in severe colitis. 


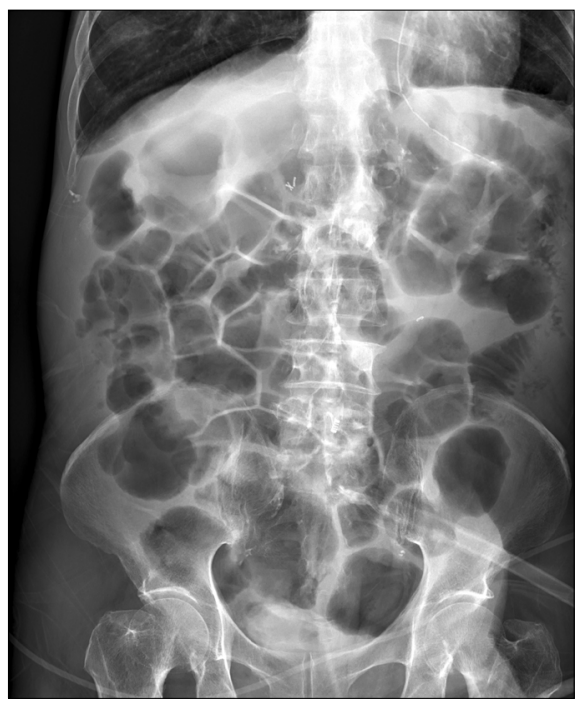

Fig. 3. Abdominal plain radiogram obtained before neostigmine administration.

softer abdomen, and continuous bowel sounds could be heard. As a result, enteral feeding was started a week after neostigmine administration, and oral feeding was initiated following observations of normal bowel movements.

In this case, the use of neostigmine under careful monitoring was safe and effective for a critically ill patient with paralytic ileus, who did not respond to conservative management.

\section{Discussion}

A number of pathophysiologic mechanisms contribute to the development of paralytic ileus. Administration of opioids or catecholamines is one of the major factors giving rise to paralytic ileus in critically ill patients. Paralytic ileus is also often associated with intraperitoneal or retroperitoneal infection, and edema or ascites secondary to massive fluid resuscitation during septic or toxic shock with capillary leakage. Diffuse inflammation of the intestinal wall due to inflammatory bowel disease, acute bacterial or parasitic intestinal infection, or antibiotic-induced pseudomembranous colitis with toxic megacolon, may lead to severe paralytic ileus. Furthermore, mesenteric ischemia, abdominal vascular injury, retroperitoneal or intrabdominal hematoma, and metabolic disturbances, such as hypokalemia, may also cause ileus. Paralytic ileus can affect critically ill patients in various ways,

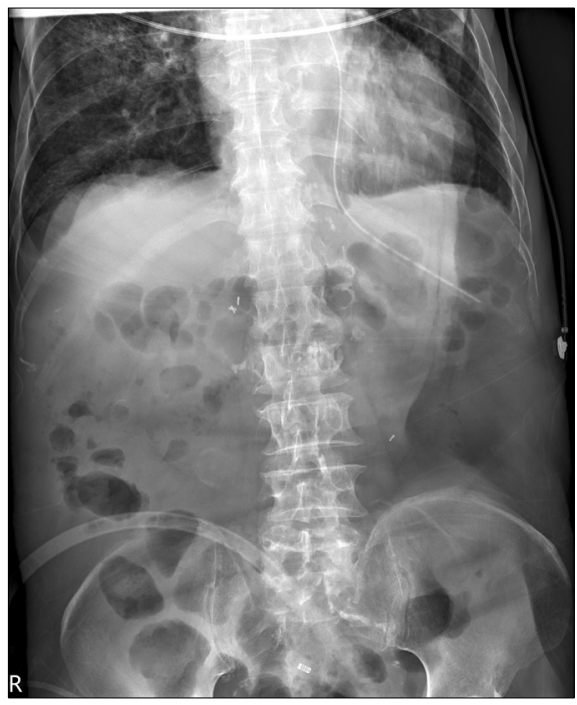

Fig. 4. Abdominal plain radiogram obtained after neostigmine administration.

including impediment of enteral nutrition (EN), increased intraabdominal pressure, bacterial hyperproliferation, injury of the intestinal mucosa, and bacterial translocation through the injured mucosa [4].

The significance of $\mathrm{EN}$ in the intensive care unit is already widely known. Conventional treatment of paralytic ileus emphasizes decompression using NPO and L-tube. However, recent guidelines recommend initiation of $\mathrm{EN}$ for patients with paralytic ileus [5]. On the other hand, impairment of motility and compromised propulsion of the intestinal contents may increase gastric residues and cause gastric regurgitation of serous fluid, and aspiration into the tracheobronchial tree. As a result, this can increase the risk of aspiration pneumonia. Moreover, intolerable EN may further exacerbate paralytic ileus. In consideration of these points, initiation of $\mathrm{EN}$ in patients with paralytic ileus is rather difficult. Inadequate nutritional support, as a consequence, may lead to a worse prognosis by reducing patients' overall muscle strength and functional capacity. This diminishes the ability to synthesize new tissues and heal wounds, increasing the number of infections, hospital stay, morbidity, and mortality [6].

Increased intraabdominal pressure interferes with contraction of the diaphragm, reduces lung compliance, and increases respiratory effort [7]. This delays weaning from mechanical ventilation 
and prevents vigorous rehabilitation. It may increase the number of days spent in ICU and slow return to everyday life. Acutely increased intraabdominal pressure may cause multiple organ failure through perfusion disturbances in various body organs [4]. Patients with ileus are subjected to bacterial translocation, which is affected by three determinants: 1) bacterial overgrowth, 2) inflammation and impairment of the barrier function of the intestinal wall, and 3) impairment of systemic immunocompetence, seen in most critically ill patients [8]. Thus, ileus can promote a systemic inflammatory response syndrome and also progress to multiple-organ dysfunction syndrome, even in the absence of major elevations in IAP [9]. Since paralytic ileus may exacerbate the clinical severity of patients in ICU, appropriate treatment must also be implemented. However, an effective treatment modality has not yet been revealed.

By interfering with acetylcholinesterase, which breaks down acetylcholine, neostigmine indirectly stimulates both nicotinic and muscarinic receptors. Neostigmine binds to the anionic and esteric sites of cholinesterase. The drug blocks the active site of acetylcholinesterase, so that the enzyme can no longer break down the acetylcholine molecules before they reach the postsynaptic membrane receptors. This increases the synaptic concentration of the neurotransmitter, enabling the postsynaptic neuron to be activated more easily. Well-known adverse effects include bradycardia, abdominal cramps, vomiting, and excessive salivation. Neostigmine is indicated for patients with myasthenia gravis to improve muscle tone, and is also used during general anesthesia to reverse the effect of a muscular relaxant. Furthermore, neostigmine is used to treat urinary retention occurring after induction of general anesthesia [3]. Neostigmine is also indicated for acute colonic pseudo-obstruction. The results of four uncontrolled studies suggest that IV administration of neostigmine causes rapid colonic decompression in patients with acute colonic pseudo-obstruction [10]. This pharmacologic approach is based on the theory that acute colonic pseudo-obstruction results from ineffectual colonic motility caused by excessive sympathetic stimulation, parasympathetic dysfunction, or both. In the premise that a similar mechanism of action may apply to paralytic ileus, this patient was administered neostigmine. As a result, ileus improved and oral feeding was recommenced for this patient before he was discharged from SICU. No adverse effects other than excessive salivation were manifested in this case. Nevertheless, a parasympathomimetic agent, such as neostigmine, may not always be safe. In particular, for patients with bradyarrhythmia or those receiving a concurrent regimen of a beta-adrenergic antagonist, neostigmine may cause fatal bradycardia or increase airway secretions to exacerbate active bronchospasm. Thus, administration of neostigmine must be accompanied by close observations under proper monitoring and hemodynamic stability, and it should be administered only to patients with a low risk of developing respiratory complications.

In summary, neostigmine can be effective in patients in ICU with paralytic ileus, which does not respond to conservative treatments. Nonetheless, administration of neostigmine should be considered only for stable patients under proper monitoring to prevent development of potentially fatal adverse effects, such as symptomatic bradycardia.

\section{Conflicts of Interest}

No potential conflict of interest relevant to this article was reported.

\section{References}

1. Malbrain MLNG. Abdominal pressure in the critically ill. Curr Opin Crit Care 2000;6:17-29.

2. Holte K, Kehlet H. Postoperative ileus: progress towards effective management. Drugs 2002;62:2603-15.

3. Schwab RS, Chapman WP. Clinical uses of neostigmine. Med Clin North Am 1947;31:1238-60.

4. Madl C, Druml W. Gastrointestinal disorders of the critically ill. Systemic consequences of ileus. Best Pract Res Clin Gastroenterol 2003;17:445-56.

5. McClave SA, Martindale RG, Vanek VW, McCarthy M, Roberts P, Taylor B, et al. Guidelines for the provision and assessment of nutrition support therapy in the adult critically ill patient: Society of Critical Care Medicine (SCCM) and American Society for Parenteral and Enteral Nutrition (A.S.P.E.N.). JPEN J Parenter Enteral Nutr 2009;33:277-316.

6. de Azevedo RP, Machado FR. Constipation in critically ill patients: much more than we imagine. Rev Bras Ter Intensiva 2013;25:73-4.

7. van der Spoel JI, Oudemans-van Straaten HM, Kuiper MA, van Roon EN, Zandstra DF, van der Voort PH. Laxation of critically ill patients with lactulose or polyethylene glycol: a 
two-center randomized, double-blind, placebo-controlled trial. Crit Care Med 2007;35:2726-31.

8. Munford RS, Pugin J. Normal responses to injury prevent systemic inflammation and can be immunosuppressive. Am J Respir Crit Care Med 2001;163:316-21.

9. Heinzelmann M, Simmen HP, Battaglia H, Friedl HP, Trentz
O. Inflammatory response after abdominal trauma, infection, or intestinal obstruction measured by oxygen radical production in peritoneal fluid. Am J Surg 1997;174:445-7.

10. Valle RG, Godoy FL. Neostigmine for acute colonic pseudoobstruction: a meta-analysis. Ann Med Surg (Lond) 2014;3: $60-4$. 